\title{
Discrete Element Modeling of Triboelectrically Charged Particles
}

\author{
Michael D. Hogue ${ }^{1}$, Carlos I. Calle ${ }^{1}$, Peter S.Weitzman ${ }^{2}$, David R. Curry ${ }^{2}$ \\ 'Electrostatics \& Surface Physics Laboratory \\ NASA, Kennedy Space Center, FL 32899 \\ Mailcode: KT-B-1 \\ e-mail: Michael.D.Hogue@nasa.gov \\ ${ }^{2}$ DEM Solutions USA Inc. \\ 20 Blueberry Meadow Lane \\ Lebanon, NH 03766, USA \\ e-mail: Pweitzman@dem-solutions.com
}

Keywords: Triboelectric, Discrete Element Modeling, Particles, Dielectrophoresis, Van der Waals Forces.

Abstract - Tribocharging of particles is common in many processes including fine powder handling and mixing, printer toner transport and dust extraction. In a lunar environment with its high vacuum and lack of water, electrostatic forces are an important factor to consider when designing and operating equipment. Dust mitigation and management is critical to safe and predictable performance of people and equipment. The extreme nature of lunar conditions makes it difficult and costly to carryout experiments on earth which are necessary to better understand how particles gather and transfer charge between each other and with equipment surfaces. DEM (Discrete Element Modeling) provides an excellent virtual laboratory for studying tribocharging of particles as well as for design of devices for dust mitigation and for other purposes related to handling and processing of lunar regolith. Theoretical and experimental work has been performed pursuant to incorporating screened Coulombic electrostatic forces into $\mathrm{EDEM}^{\mathrm{TM}}$, a commercial DEM software package. The DEM software is used to model the trajectories of large numbers of particles for industrial particulate handling and processing applications and can be coupled with other solvers and numerical models to calculate particle interaction with surrounding media and force fields.

While simple Coulombic force between two particles is well understood, its operation in an ensemble of particles is more complex. When the tribocharging of particles and surfaces due to frictional contact is also considered, it is necessary to consider longer range of interaction of particles in response to electrostatic charging. The standard DEM algorithm accounts for particle mechanical properties and inertia as a function of particle shape and mass. If fluid drag is neglected, then particle dynamics are governed by contact between particles, between particles and equipment surfaces and gravity forces. Consideration of particle charge and any tribocharging and electric field effects requires calculation of the forces due to these effects.

Body forces such as an electrostatic force can be added during the DEM calculational cycle as shown in Fig 1. The DEM software provides a means of calling routines to calculate body forces acting over a short time increment and then adding these forces to 
those calculated for physical contact. The electrostatic forces due to the triboelectrification of the particles were added using an external model which also took into account the presence of neighboring particles which act to screen the charge between particles over a distance.

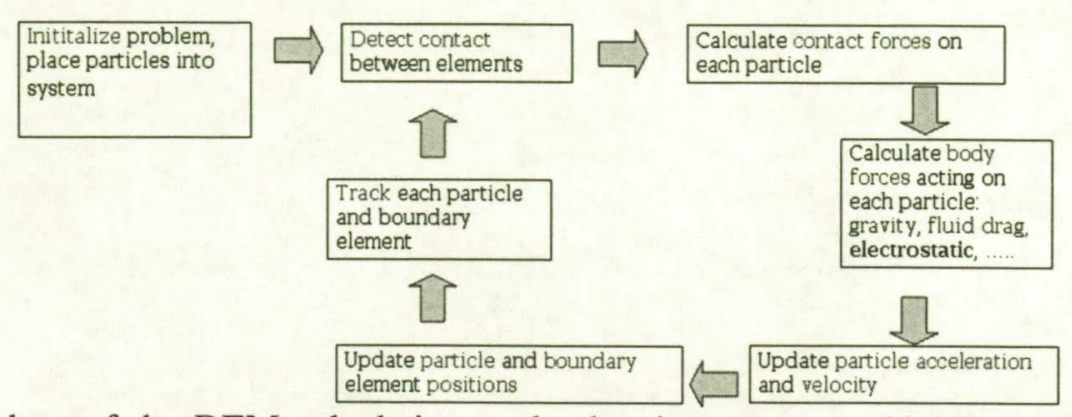

Fig 1 Flowchart of the DEM calculation cycle showing stage at which body forces such as electrostatic forces are calculated and added to the computed contact forces before updating the particle acceleration and integrating for new velocity and position over a timestep.

The screened Coulombic force is given by

$$
F_{S}=-\frac{d U_{e}}{d r}=\frac{q_{1} q_{2}}{4 \pi \varepsilon_{0}}\left(\frac{\kappa}{r}+\frac{1}{r^{2}}\right) e^{-\kappa r},
$$

where $U_{e}$ is the electrostatic potential, $\kappa$ is the inverse Debye length which is dependent on particle concentration, $q_{1}$ and $q_{2}$ are the charges on particles 1 and $2, \varepsilon_{0}$ is the electric permittivity of free space, and $r$ is the distance between particle centers.

To determine triboelectric charging of the particles, a charge generation equation was used

$$
q(t)=q_{s}\left(1-e^{-\alpha t}\right)
$$

where $q(t)$ is the charge generated on the particle at time $t, q_{s}$ is the saturation charge, and $\alpha$ is the material dependent charge generation constant [1]

This paper presents an overview of the theory, results of experiment and comparison to the results of the DEM simulations. The interaction of charged particles with charged (and uncharged) surfaces such as handling machinery or structures is investigated. Issues are also discussed related to refining the current model by addition of other mechanical forces such as dynamic friction for translations and rotations which influence particle dynamics as well as other electrostatic phenomena such as the dielectrophoretic and Van der Waals forces.

Reference:

[1] W. D. Greason, "Investigation of a test methodology for triboelectrification", Jour. Electrostat. 49, Issue 3-4 (Aug, 2000) pp. 245-256. 